DOI: $10.2478 /$ ausm-2014-0016

\title{
Hydromagnetic thermosolutal instability of Rivlin-Ericksen rotating fluid permeated with suspended particles and variable gravity field in porous medium
}

\author{
G. C. Rana \\ Department of Mathematics NSCBM Govt. P.G. \\ College, Hamirpur-177005, Himachal Pradesh, India \\ email: drgcrana15@gmail.com
}

\begin{abstract}
The thermosolutal instability of Rivlin-Ericksen elasticoviscous rotating fluid permeated with suspended particles (fine dust) and variable gravity field in porous medium in hydromagnetics is considered. By applying normal mode analysis method, the dispersion relation has been derived and solved analytically. It is observed that the rotation, magnetic field, gravity field, suspended particles and viscoelasticity introduce oscillatory modes. For stationary convection, the rotation and stable solute gradient has stabilizing effects and suspended particles are found to have destabilizing effect on the system whereas the medium permeability has stabilizing or destabilizing effect on the system under certain conditions. The magnetic field has destabilizing effect in the absence of rotation whereas in the presence of rotation, magnetic field has stabilizing or destabilizing effect under certain conditions. The effect of rotation, suspended particles, magnetic field, stable solute gradient and medium permeability has also been shown graphically.
\end{abstract}

\section{Introduction}

A detailed account of the thermal instability of a Newtonian fluid, under varying assumptions of hydrodynamics and hydromagnetics has been given by

2010 Mathematics Subject Classification: 76A05, 76A10, 76E07, 76S05

Key words and phrases: Rivlin-Ericksen fluid, thermosolutal instability, suspended particles, magnetic field, variable gravity field, porous medium 
Chandrasekhar [3]. Bhatia and Steiner [1] have studied the thermal instability of a Maxwellian visco-elastic fluid in the presence of magnetic field while the thermal convection in Oldroydian visco-elastic fluid has been considered by Sharma [14]. Veronis [20] has investigated the problem of thermohaline convection in a layer of fluid heated from below and subjected to a stable salinity gradient. The buoyancy forces can arise not only from density differences due to variations in solute concentration. Thermosolutal instability problems arise in oceanography, limnology and engineering.

The medium has been considered to be non-porous in all the above studies. Lapwood [5] has studied the convective flow in a porous medium using linearized stability theory. The Rayleigh instability of a thermal boundary layer in flow through a porous medium has been considered by Wooding [21] whereas Scanlon and Segel [13] have considered the effect of suspended particles on the onset of Be'nard convection and found that the critical Rayleigh number was reduced solely because the heat capacity of the pure gas was supplemented by the particles. The suspended particles were thus found to destabilize the layer.

Sharma and Sunil [15] have studied the thermal instability of an Oldroydian viscoelastic fluid with suspended particles in hydromagnetics in a porous medium. There are many elastico-viscous fluids that cannot be characterized by Maxwell's constitutive relations or Oldroyd's constitutive relations. One such class of fluids is Rivlin-Ericksen [12] elastico-viscous fluid. Srivastava and Singh [18] have studied the unsteady flow of a dusty elastico-viscous RivlinEricksen fluid through channels of different cross-sections in the presence of time-dependent pressure gradient. Garg et al. [4] has studied the rectilinear oscillations of a sphere along its diameter in conducting dusty Rivlin-Ericksen fluid in the presence of magnetic field.

Stommel and Fedorov [19] and Linden [6] have remarked that the length scalar characteristic of double diffusive convecting layers in the ocean may be sufficiently large that the Earth's rotation might be important in their formation. Moreover, the rotation of the Earth distorts the boundaries of a hexagonal convection cell in a fluid through a porous medium and the distortion plays an important role in the extraction of energy in the geothermal regions. Brakke [2] explained a double-diffusive instability that occurs when a solution of a slowly diffusing protein is layered over a denser solution of more rapidly diffusing sucrose. The problem of thermosolutal convection in fluids in a porous medium is of importance in geophysics, soil sciences, ground water hydrology and astrophysics. The scientific importance of the field has also increased because hydrothermal circulation is the dominant heat transfer mechanism in the development of young oceanic crust (Lister, [7]). Gener- 
ally, it is accepted that comets consist of a dusty 'snowball' of a mixture of frozen gases which in the process of their journey change from solid to gas and vice-versa. The physical properties of comets, meteorites and inter-planetary dust strongly suggest the importance of porosity in the astrophysical context (McDonnel, [8]).

Thermal instability of a fluid layer under variable gravitational field heated from below or above is investigated analytically by Pradhan and Samal [9]. Although the gravity field of the Earth is varying with height from its surface, we usually neglect this variation for laboratory purposes and treat the field as constant. However, this may not the case for large scale flows in the ocean, the atmosphere or the mantle. It can become imperative to consider gravity as a quantity varying with distance from the centre.

A porous medium is a solid with holes in it, and is characterized by the manner in which the holes are imbedded, how they are interconnected and the description of their location, shape and interconnection. However, the flow of a fluid through a homogeneous and isotropic porous medium is governed by Darcy's law which states that the usual viscous term in the equations of motion of Rivlin-Ericksen fluid is replaced by the resistance term $\left[-\frac{1}{k_{1}}\left(\mu+\mu^{\prime} \frac{\partial}{\partial t}\right)\right] \mathbf{q}$, where $\mu$ and $\mu^{\prime}$ are the viscosity and viscoelasticity of the incompressible Rivlin-Ericksen fluid, $k_{1}$ is the medium permeability and $q$ is the Darcian (filter) velocity of the fluid (Garg et al. [4], Sharma and Sunil [15] and Sharma and Rana $[16,17])$.

Sharma and Rana [16] have studied thermal instability of Walters' (Model $\mathrm{B}^{\prime}$ ) elastico-viscous in the presence of variable gravity field and rotation in porous medium. Sharma and Rana [17] have also studied the thermosolutal instability of incompressible Walters' (Model B') rotating fluid permeated with suspended particles and variable gravity field in porous medium. Recently, Rana and Kumar [11] have studied thermal instability of RivlinEricksen elastico-viscous rotating fluid permeated with suspended particles and variable gravity field in porous medium and thermal instability of compressible Walters' (Model B') elastico-viscous rotating fluid a permeated with suspended dust particles in porous medium have been studied by Rana and Kango [10]. Keeping in mind the importance in various applications mentioned above, our interest, in the present paper is to study the thermosolutal instability of Rivlin-Ericksen elastico-viscous rotating fluid permeated with suspended particles and variable field in porous medium in hydromagnetics. 


\section{Mathematical formulation of the problem}

Consider an infinite horizontal layer of an electrically conducting Rivlin-Ericksen elastico-viscous fluid of depth $\mathrm{d}$ in a porous medium bounded by the planes $z=0$ and $z=\mathrm{d}$ in an isotropic and homogeneous medium of porosity $\epsilon$ and permeability $k_{1}$, which is acted upon by a uniform rotation $\Omega(0,0, \Omega)$ uniform vertical magnetic field $\mathbf{H}(0,0, \mathbf{H})$ and variable gravity $\mathbf{g}(0,0,-\mathrm{g}), \mathrm{g}=\lambda \mathrm{g}_{0}$, $g_{0}(>0)$ is the value of $g$ at $z=0$ and $\lambda$ can be positive or negative as gravity increases or decreases upward from its value go. This layer is heated and soluted from below such that a uniform temperature gradient $\beta\left(=\left|\frac{\mathrm{dT}}{\mathrm{d} z}\right|\right)$ and a uniform solute gradient $\beta^{\prime}\left(=\left|\frac{\mathrm{dC}}{\mathrm{d} z}\right|\right)$ are maintained as shown in schematic sketch of physical situation.

The character of equilibrium of this initial static state is determined by supposing that the system is slightly disturbed and then following its further evolution.

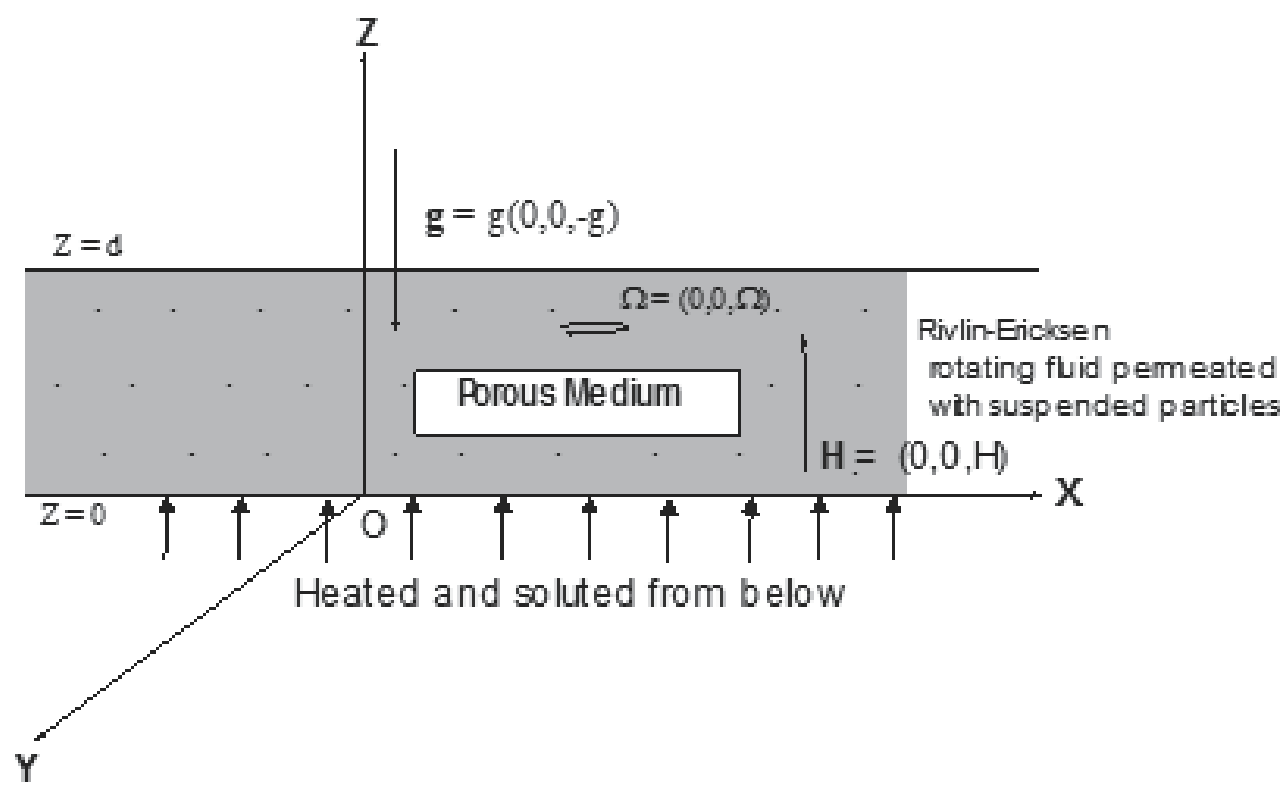

Schem atic Sketch of Physical Situation 
The hydromagnetic equations in porous medium (Chandrasekhar [3], Rivlin and Ericksen [12], Rana and Kumar [11]) relevant to the problem are

$$
\begin{aligned}
& \frac{1}{\epsilon}\left[\frac{\partial \mathbf{q}}{\partial t}+\frac{1}{\epsilon}(\mathbf{q} \cdot \nabla) \mathbf{q}\right]=-\frac{1}{\rho_{0}} \nabla p+g\left(1+\frac{\delta \rho}{\rho_{0}}\right)-\frac{1}{k_{1}}\left(v+v^{\prime} \frac{\partial}{\partial t}\right) \mathbf{q} \\
& \quad+\frac{2}{\epsilon}(\mathbf{q} \times \Omega)+\frac{K^{\prime} N}{\rho_{0} \epsilon}\left(\mathbf{q}_{\mathbf{d}}-\mathbf{q}\right)+\frac{\mu_{e}}{4 \pi \rho_{0}}(\nabla \times \mathbf{H}) \times \mathbf{H}, \\
& \nabla \cdot \mathbf{q}=0, \\
& \mathrm{E} \frac{\partial \mathrm{T}}{\partial \mathrm{t}}+(\mathbf{q} \cdot \nabla) \mathrm{T}+\frac{\mathrm{mNC}_{\mathrm{pt}}}{\rho_{0} \mathrm{C}_{\mathrm{f}}}\left[\epsilon \frac{\partial}{\partial t}+\mathbf{q}_{\mathbf{d}} \cdot \nabla\right] \mathrm{T}=\kappa \nabla^{2} \mathrm{~T}, \\
& \mathrm{E}^{\prime} \frac{\partial \mathrm{C}}{\partial \mathrm{t}}+(\mathbf{q} \cdot \nabla) \mathrm{T}+\frac{\mathrm{mNC}_{\mathrm{pt}}^{\prime}}{\rho_{0} \mathrm{C}_{\mathrm{f}}^{\prime}}\left[\epsilon \frac{\partial}{\partial t}+\mathbf{q}_{\mathbf{d}} \cdot \nabla\right] \mathrm{T}=\kappa^{\prime} \nabla^{2} \mathrm{~T} \\
& \nabla \cdot \mathbf{H}=0, \\
& \epsilon \frac{\partial \mathbf{H}}{\partial \mathrm{t}}=\nabla \times(\mathbf{q} \times \mathbf{H})+\epsilon \eta \nabla^{2} \mathbf{H},
\end{aligned}
$$

where $E=\epsilon+(1-\epsilon)\left(\frac{\rho_{s} c_{s}}{\rho_{0} c_{f}}\right), \rho_{s}, c_{s} ; \rho_{0}, c_{f}$ denote the density and heat capacity of solid (porous) matrix and fluid respectively and $E^{\prime}$ is a constant analogous to $E$ but corresponding to solute rather than heat; $\kappa, \kappa^{\prime}$ are the thermal diffusivity and solute diffusivity respectively.

The equation of state is

$$
\rho=\rho_{0}\left[1-\alpha\left(T-T_{0}\right)+\alpha^{\prime}\left(C-C_{0}\right)\right],
$$

where the suffix zero refers to values at the reference level $z=0$. Here $\rho, v, v^{\prime}$, $p, \epsilon, T, C, \mu_{e}, \alpha, \alpha^{\prime}, \mathbf{q}(0,0,0)$ and $\mathbf{H}(0,0, H)$ stand for density, kinematic viscosity, kinematic viscoelasticity, pressure, medium porosity, temperature, solute concentration, magnetic permeability, thermal coefficient of expansion, an analogous solvent coefficient of expansion, velocity of the fluid and magnetic field. Here $\mathbf{q}_{\mathbf{d}}(\bar{x}, t)$ and $N(\bar{x}, t)$ denote the velocity and number density of the particles respectively, $K=6 \pi \eta \rho v$, where $\eta$ is particle radius, is the Stokes drag coefficient, $\mathbf{q}_{\mathbf{d}}=(l, r, s)$ and $\bar{x}=(x, y, z)$.

If $\mathrm{mN}$ is the mass of particles per unit volume, then the equations of motion and continuity for the particles are

$$
\begin{aligned}
& \mathrm{mN}\left[\frac{\partial \mathbf{q}_{\mathbf{d}}}{\partial t}+\frac{1}{\epsilon}\left(\mathbf{q}_{\mathbf{d}} \cdot \nabla\right) \mathbf{q}_{\mathbf{d}}\right]=\mathrm{K}^{\prime} \mathrm{N}\left(\mathbf{q}-\mathbf{q}_{\mathbf{d}}\right), \\
& \epsilon \frac{\partial \mathrm{N}}{\partial \mathrm{t}}+\nabla \cdot\left(\mathrm{Nq}_{\mathbf{d}}\right)=0 .
\end{aligned}
$$


The presence of particles adds an extra force term proportional to the velocity difference between particles and fluid and appears in the equation of motion (1). Since the force exerted by the fluid on the particles is equal and opposite to that exerted by the particles on the fluid, there must be an extra force term, equal in magnitude but opposite in sign, in the equations of motion for the particles (8). The buoyancy force on the particles is neglected. Interparticles reactions are not considered either since we assume that the distance between the particles are quite large compared with their diameters. These assumptions have been used in writing the equations of motion (8) for the particles.

The initial state of the system is taken to be quiescent layer (no settling) with a uniform particle distribution number. The initial state is

$$
\begin{gathered}
\mathbf{q}=(0,0,0), \mathbf{q}_{\mathbf{d}}=(0,0,0), \\
T=-\beta z+\mathrm{T}_{0}, C=-\beta^{\prime} z+\mathrm{C}_{0}, \\
\rho=\rho_{0}\left(1+\alpha \beta z-\alpha^{\prime} \beta^{\prime} z\right), \mathrm{N}_{0}=\mathrm{constant}
\end{gathered}
$$

is an exact solution to the governing equations.

\section{Perturbation equations}

Let $\mathbf{q}(u, v, w), \mathbf{q}_{d}(l, r, s), \theta, \gamma, \delta p$ and $\delta \rho$ denote, respectively, the perturbations in fluid velocity $\mathbf{q}(0,0,0)$, the perturbation in particle velocity $\mathbf{q}_{d}(0,0,0)$, temperature $\mathrm{T}$, solute concentration $\mathrm{C}$, pressure p and density $\rho$.

The change in density $\delta \rho$ caused by perturbation of temperature $\theta$ and solute concentration $\gamma$ is given by

$$
\delta \rho=-\rho_{0}\left(\alpha \theta-\alpha^{\prime} \gamma\right)
$$

The linearized perturbation equations governing the motion of fluids are

$$
\begin{aligned}
& \frac{1}{\epsilon} \frac{\partial \mathbf{q}}{\partial t}=-\frac{1}{\rho_{0}} \Omega \delta p-g\left(\alpha \theta-\alpha^{\prime} \gamma\right)-\frac{1}{k_{1}}\left(v+v^{\prime} \frac{\partial}{\partial t}\right) q \\
& +\frac{K^{\prime} N}{\epsilon}\left(\mathbf{q}_{\mathbf{d}}-\mathbf{q}\right)+\frac{2}{\epsilon}(\mathbf{q} \times \Omega)+\frac{\mu_{e}}{4 \pi \rho_{0}}(\nabla \times \mathbf{h}) \times \mathbf{H}, \\
& \nabla \cdot \mathbf{q}=0 \\
& \left(\frac{m}{K^{\prime}} \frac{\partial}{\partial t}+1\right) q_{d}=q \text {, }
\end{aligned}
$$




$$
\begin{aligned}
& (E+b \epsilon) \frac{\Omega \theta}{\partial t}=\beta(w+b s)+\kappa \nabla^{2} \theta, \\
& \left(E^{\prime}+b^{\prime} \epsilon\right) \frac{\Omega \theta}{\partial t}=\beta^{\prime}\left(w+b^{\prime} s\right)+\kappa^{\prime} \nabla^{2} \gamma \\
& \nabla \cdot \mathbf{h}=0, \\
& \epsilon \frac{\partial \mathbf{H}}{\partial t}=(\mathbf{H} \cdot \nabla) q+\epsilon \eta \nabla^{2} \mathbf{H},
\end{aligned}
$$

where $b=\frac{m N C_{p t}}{\rho_{0} C_{f}}, b^{\prime}=\frac{m N C_{p t}^{\prime}}{\rho_{0} C_{f}^{\prime}}$ and $w, s$ are the vertical fluid and particles velocity.

In the Cartesian form, equations (12)-(18) can be expressed as

$$
\begin{aligned}
& \frac{1}{\epsilon}\left(\frac{m}{K^{\prime}} \frac{\partial}{\partial t}+1\right) \frac{\partial u}{\partial t}=-\frac{1}{\rho_{0}}\left(\frac{m}{K^{\prime}} \frac{\partial}{\partial t}+1\right) \frac{\partial}{\partial x}(\delta p) \\
& -\frac{1}{k_{1}}\left(v+v^{\prime} \frac{\partial}{\partial t}\right)\left(\frac{m}{K^{\prime}} \frac{\partial}{\partial t}+1\right) u-\frac{m N}{\epsilon \rho_{0}} \frac{\partial u}{\partial t} \\
& +\frac{\mu_{e} H}{4 \pi \rho_{0}}\left(\frac{\partial h_{x}}{\partial z}-\frac{\partial h_{z}}{\partial x}\right)+\frac{2}{\epsilon}\left(\frac{m}{K^{\prime}} \frac{\partial}{\partial t}+1\right) \Omega v, \\
& \frac{1}{\epsilon}\left(\frac{m}{K^{\prime}} \frac{\partial}{\partial t}+1\right) \frac{\partial v}{\partial t}=-\frac{1}{\rho_{0}}\left(\frac{m}{K^{\prime}} \frac{\partial}{\partial t}+1\right) \frac{\partial}{\partial y}(\delta p) \\
& -\frac{1}{k_{1}}\left(v+v^{\prime} \frac{\partial}{\partial t}\right)\left(\frac{m}{K^{\prime}} \frac{\partial}{\partial t}+1\right) v-\frac{m N}{\epsilon \rho_{0}} \frac{\partial v}{\partial t} \\
& +\frac{\mu_{e} H}{4 \pi \rho_{0}}\left(\frac{\partial h_{y}}{\partial z}-\frac{\partial h_{z}}{\partial y}\right) \frac{2}{\epsilon}\left(\frac{m}{K^{\prime}} \frac{\partial}{\partial t}+1\right) \Omega u, \\
& \frac{1}{\epsilon}\left(\frac{m}{K^{\prime}} \frac{\partial}{\partial t}+1\right) \frac{\partial w}{\partial t}=-\frac{1}{\rho_{0}}\left(\frac{m}{K^{\prime}} \frac{\partial}{\partial t}+1\right) \frac{\partial}{\partial z}(\delta p)-\frac{1}{k_{1}}\left(v+v^{\prime} \frac{\partial}{\partial t}\right) \\
& \left(\frac{m}{K^{\prime}} \frac{\partial}{\partial t}+1\right) w-\frac{m N}{\epsilon \rho_{0}} \frac{\partial w}{\partial t}+g\left(\frac{m}{K^{\prime}} \frac{\partial}{\partial t}+1\right) \alpha \theta, \\
& \frac{\partial u}{\partial x}+\frac{\partial v}{\partial y}+\frac{\partial w}{\partial z}=0 \text {, } \\
& (\mathrm{E}+\mathrm{b} \epsilon) \frac{\Omega \theta}{\partial \mathrm{t}}=\beta(w+\mathrm{bs})+\kappa \nabla^{2} \theta, \\
& \left(E^{\prime}+b^{\prime} \epsilon\right) \frac{\Omega \theta}{\partial t}=\beta^{\prime}\left(w+b^{\prime} s\right)+\kappa^{\prime} \nabla^{2} \gamma \\
& \epsilon \frac{\partial h_{x}}{\partial t}=H \frac{\partial u}{\partial z}+\epsilon \eta \nabla^{2} h_{x},
\end{aligned}
$$




$$
\begin{aligned}
& \epsilon \frac{\partial h_{y}}{\partial t}=H \frac{\partial v}{\partial z}+\epsilon \eta \nabla^{2} h_{y}, \\
& \epsilon \frac{\partial h_{z}}{\partial t}=H \frac{\partial w}{\partial z}+\epsilon \eta \nabla^{2} h_{z}, \\
& \frac{\partial h_{x}}{\partial x}+\frac{\partial h_{y}}{\partial y}+\frac{\partial h_{z}}{\partial z}=0 .
\end{aligned}
$$

Operating equation (19) and (20) by $\frac{\partial}{\partial x}$ and $\frac{\partial}{\partial y}$ respectively, adding and using equation (25)-(28), we get

$$
\begin{gathered}
\frac{1}{\epsilon}\left(\frac{m}{K^{\prime}} \frac{\partial}{\partial t}+1\right) \frac{\partial}{\partial t}\left(\frac{\partial w}{\partial z}\right)=\frac{1}{\rho_{0}}\left(\frac{m}{K^{\prime}} \frac{\partial}{\partial t}+1\right)\left(\nabla^{2}-\frac{\partial^{2}}{\partial z^{2}}\right) \delta p- \\
-\frac{1}{k_{1}}\left(v+v^{\prime} \frac{\partial}{\partial t}\right)\left(\frac{m}{K^{\prime}} \frac{\partial}{\partial t}+1\right)\left(\frac{\partial w}{\partial z}\right)-\frac{m N}{\epsilon \rho_{0}} \frac{\partial}{\partial t}\left(\frac{\partial w}{\partial z}\right)+ \\
\quad+\left(\frac{m}{K^{\prime}} \frac{\partial}{\partial t}+1\right) \frac{e}{4 \pi \rho_{0}} \nabla^{2} h_{z}-\frac{2}{\epsilon}\left(\frac{m}{K^{\prime}} \frac{\partial}{\partial t}+1\right) \Omega \zeta,
\end{gathered}
$$

where $\zeta=\frac{\partial v}{\partial x}-\frac{\partial u}{\partial y}$ is the z-component of vorticity.

Operating equation (21) and (29) by $\left(\nabla^{2}-\frac{\partial^{2}}{\partial z^{2}}\right)$ and $\frac{\partial}{\partial z}$ respectively and adding to eliminate $\delta p$ between equations (21) and (29), we get

$$
\begin{aligned}
& \frac{1}{\epsilon}\left(\frac{m}{K^{\prime}} \frac{\partial}{\partial t}+1\right) \frac{\partial}{\partial t}\left(\nabla^{2} w\right)-\frac{1}{k_{1}}\left(v-v^{\prime} \frac{\partial}{\partial t}\right)\left(\frac{m}{K^{\prime}} \frac{\partial}{\partial t}+1\right) \nabla^{2} w+ \\
& +g\left(\frac{\partial^{2}}{\partial x^{2}}+\frac{\partial^{2}}{\partial y^{2}}\right)\left(\frac{m}{K^{\prime}} \frac{\partial}{\partial t}+1\right) \alpha \theta-\frac{m N}{\epsilon \rho_{0}} \frac{\partial}{\partial t}\left(\nabla^{2} w\right)+ \\
& \quad+\frac{e}{4 \pi \rho_{0}}\left(\frac{m}{K^{\prime}} \frac{\partial}{\partial t}+1\right) \frac{\partial}{\partial t} \nabla^{2} h_{z}-\frac{2}{\epsilon}\left(\frac{m}{K^{\prime}} \frac{\partial}{\partial t}+1\right) \Omega \frac{\Omega \zeta}{\partial z},
\end{aligned}
$$

where $\nabla^{2}=\frac{\partial^{2}}{\partial x^{2}}+\frac{\partial^{2}}{\partial y^{2}}+\frac{\partial^{2}}{\partial z^{2}}$.

Operating equation (19) and (20) by $-\frac{\partial}{\partial y}$ and $\frac{\partial}{\partial x}$ respectively and adding, we get

$$
\begin{aligned}
& \frac{1}{\epsilon}\left(\frac{m}{K^{\prime}} \frac{\partial}{\partial t}+1\right) \frac{\Omega \zeta}{\partial t}=-\frac{1}{k_{1}}\left(v-v^{\prime} \frac{\partial}{\partial t}\right)\left(\frac{m}{K^{\prime}} \frac{\partial}{\partial t}+1\right) \zeta- \\
& \quad-\frac{m N}{\epsilon \rho_{0}} \frac{\Omega \zeta}{\partial t}+\frac{2}{\epsilon}\left(\frac{m}{K^{\prime}} \frac{\partial}{\partial t}+1\right) \Omega \frac{\partial w}{\partial z}+\frac{\mu_{e} H}{4 \pi \rho_{0}}\left(\frac{m}{K^{\prime}} \frac{\partial}{\partial t}+1\right) \frac{\Omega \xi}{\partial t},
\end{aligned}
$$

where $\xi=\frac{\partial h_{y}}{\partial x}-\frac{\partial h_{x}}{\partial y}$ is the $z$-component of current density. 
Operating equations (25) and (26) by $-\frac{\partial}{\partial y}$ and $\frac{\partial}{\partial x}$ respectively and adding, we get

$$
\frac{1}{\epsilon} \frac{\Omega \xi}{\partial t}=H \frac{\Omega \xi}{\partial t}+\epsilon \eta \nabla^{2} \xi
$$

\section{Dispersion relation}

Analyzing the disturbances into normal modes, we assume that the perturbation quantities have $\mathrm{x}, \mathrm{y}$ and $\mathrm{t}$ dependence of the form

$$
\begin{gathered}
{\left[w, s, \theta, \gamma, \zeta, h_{z}, \xi\right]=[W(z), S(z), \Theta(z), Z(z), \Gamma(z), K(z), X(z)]} \\
\exp \left(i k_{x} x+i k_{y} y+n t\right)
\end{gathered}
$$

where $k_{x}$ and $k_{y}$ are the wave numbers in the $x$ and $y$ directions, $k=\left(k_{x}{ }^{2}+k_{y}{ }^{2}\right)^{1 / 2}$ is the resultant wave number and $n$ is the frequency of the harmonic disturbance, which is, in general, a complex constant.

Using expression (33) in (30)-(32), (27), (23), and (24) become

$$
\begin{gathered}
\frac{n}{\epsilon}\left[\frac{d^{2}}{d z^{2}}-k^{2}\right] W=-g k^{2}\left(\alpha \Theta-\alpha^{\prime} \Gamma\right)-\frac{1}{k_{1}}\left(v+v^{\prime} n\right)\left(\frac{d^{2}}{d z^{2}}-k^{2}\right) W \\
-\frac{m N n}{\epsilon \rho_{0}\left(\frac{m}{k^{\prime}} n+1\right)}\left(\frac{d^{2}}{d z^{2}}-k^{2}\right) W-\frac{2 \Omega}{\epsilon} \frac{d Z}{d z}+\frac{\mu_{e} H}{4 \pi \rho_{0}} \frac{d}{d z}\left(\frac{d^{2}}{d z^{2}}-k^{2}\right) K \\
\frac{n}{\epsilon} Z=-\frac{1}{k_{1}}\left(v+v^{\prime} n\right)-\frac{m N n}{\epsilon \rho_{0}\left(\frac{m}{k^{\prime}} n+1\right)} Z+\frac{2 \Omega}{\epsilon} \frac{d W}{d z}+\frac{e^{H}}{4 \pi \rho_{0}} D X \\
\epsilon n X=H \frac{d Z}{d z}+\epsilon \eta\left(\frac{d^{2}}{d z^{2}}-k^{2}\right) X \\
\epsilon n K=H \frac{d W}{d z}+\epsilon \eta\left(\frac{d^{2}}{d z^{2}}-k^{2}\right) k \\
(E+b \epsilon) n \Theta=\beta(W+b S)+\kappa\left(\frac{d^{2}}{d z^{2}}-k^{2}\right) \Theta \\
\left(E^{\prime}+b^{\prime} \epsilon\right) n \Gamma=\beta^{\prime}\left(W+b^{\prime} S\right)+k^{\prime}\left(\frac{d^{2}}{d z^{2}}-k^{2}\right) \Gamma .
\end{gathered}
$$


Equations (34)-(39) are in non dimensional form, become

$$
\begin{aligned}
& {\left[\frac{\sigma}{\epsilon}\left(1+\frac{M}{1+\tau_{1} \sigma}\right)+\frac{1+F \sigma}{P_{l}}\right]\left(D^{2}-a^{2}\right) W+\frac{g a^{2} d^{2} \alpha \Theta}{v}-} \\
& -\frac{g a^{2} d^{2} \alpha^{\prime} \Gamma}{v}+\frac{2 \Omega d^{3}}{\epsilon v} D Z-\frac{\mu_{e} H d}{4 \pi v \rho_{0}}\left(D^{2}-a^{2}\right) D K=0, \\
& {\left[\mathrm{D}^{2}-\mathrm{a}^{2}-\mathrm{p}_{1} \sigma\right] \mathrm{X}=-\left(\frac{\mathrm{Hd}}{\epsilon \eta}\right) \mathrm{DZ},} \\
& {\left[\mathrm{D}^{2}-\mathrm{a}^{2}-\mathrm{p}_{2} \sigma\right] \mathrm{K}=-\left(\frac{\mathrm{Hd}}{\epsilon \eta}\right) \mathrm{DW},} \\
& {\left[\frac{\sigma}{\epsilon}\left(1+\frac{M}{1+\tau_{1} \sigma}\right)+\frac{1+F \sigma}{P_{l}}\right] Z=\left(\frac{2 \Omega d^{2}}{\epsilon v}\right) D W+\frac{e^{H d}}{4 \pi v \rho_{0}} D X,} \\
& {\left[D^{2}-a^{2}-E_{1} p_{1} \sigma\right] \Theta=-\left(\frac{\beta d^{2}}{\kappa}\right)\left(\frac{B+\tau_{1} \sigma}{1+\tau_{1} \sigma}\right) W} \\
& {\left[D^{2}-a^{2}-E_{1}^{\prime} p_{1}^{\prime} \sigma\right] \Gamma=-\left(\frac{\beta^{\prime} d^{2}}{k^{\prime}}\right)\left(\frac{B^{\prime}+\tau_{1} \sigma}{1+\tau_{1} \sigma}\right) W,}
\end{aligned}
$$

where we have put

$$
a=k d, \sigma=\frac{n d^{2}}{v}, \tau=\frac{m}{K^{\prime}}, \tau_{1}=\frac{\tau v}{d^{2}}, M=\frac{m N}{\rho_{0}},
$$

$E_{1}=E+b \epsilon, B=b+1, F=\frac{v^{\prime}}{d^{2}}, P_{l}=\frac{k_{1}}{d^{2}}$ is the dimensionless medium permeability, $p_{1}=\frac{v}{k}$ is the thermal Prandtl number, $p_{1}=\frac{v}{\kappa^{\prime}}$ is the Schmidt number, $\mathrm{p}_{2}=\frac{v}{\eta}$ is the magnetic Prandtl number and $\mathrm{D}^{*}=\mathrm{d} \frac{\mathrm{d}}{\mathrm{d} z}$ and the superscript $*$ is suppressed.

Applying the operator $\left(D^{2}-a^{2}-p_{2} \sigma\right)$ to the equation (41) to eliminate $X$ between equations (41) and (42), we get

$$
\begin{aligned}
& \left\{\left[\frac{\sigma}{\epsilon}\left(1+\frac{M}{1+\tau_{1} \sigma}\right)+\frac{1+F \sigma}{P_{l}}\right]\left(D^{2}-a^{2}-p_{2} \sigma\right)+\frac{Q}{\epsilon} D^{2}\right\} W \\
& \quad=\frac{2 \Omega d^{2}}{v}\left(D^{2}-a^{2}-p_{2} \sigma\right) D W .
\end{aligned}
$$


Eliminating $\mathrm{K}, \Theta$ and $Z$ between equations (40)-(46), we obtain

$$
\begin{aligned}
& {\left[\frac{\sigma}{\epsilon}\left(1+\frac{M}{1+\tau_{1} \sigma}\right)+\frac{1+F \sigma}{P_{l}}\right]\left(D^{2}-a^{2}\right)\left(D^{2}-a^{2}-E_{1} p_{1} \sigma\right)} \\
& \left(D^{2}-a^{2}-p_{2} \sigma\right)\left(D^{2}-a^{2}-E_{1}^{\prime} p_{1}^{\prime} \sigma\right) W-R a^{2} \lambda\left(\frac{B+\tau_{1} \sigma}{1+\tau_{1} \sigma}\right) \\
& \left(D^{2}-a^{2}-E_{1}^{\prime} p_{1}^{\prime} \sigma\right)\left(D^{2}-a^{2}-p_{2} \sigma\right) W+S a^{2} \lambda\left(\frac{B^{\prime}+\tau_{1} \sigma}{1+\tau_{1} \sigma}\right) \\
& \left(D^{2}-a^{2}-E_{1} p_{1} \sigma\right)\left(D^{2}-a^{2}-p_{2} \sigma\right) W+\frac{Q}{\epsilon} \\
& \left(D^{2}-a^{2}\right)\left(D^{2}-a^{2}-E_{1}^{\prime} p_{1}^{\prime} \sigma\right)\left(D^{2}-a^{2}-E_{1} p_{1} \sigma\right) W+ \\
& +\left[\frac{\frac{T_{A}}{\epsilon^{2}}\left(D^{2}-a^{2}-E_{1} p_{1} \sigma\right)\left(D^{2}-a^{2}-E_{1}^{\prime} p_{1}^{\prime} \sigma\right)\left(D^{2}-a^{2}-p_{2} \sigma\right)^{2}}{\left[\frac{\sigma}{\epsilon}\left(1+\frac{M}{1+\tau_{1} \sigma}\right)+\frac{1+F \sigma}{P_{l}}\right]\left(D^{2}-a^{2}-p_{2} \sigma\right)+\frac{Q}{\epsilon} D^{2}}\right] D^{2} W=0,
\end{aligned}
$$

where $R=\frac{g_{0} \alpha \beta d^{4}}{v k}$, is the thermal Rayleigh number,

$S=\frac{g_{0} \alpha^{\prime} \beta^{\prime} d^{4}}{v k^{\prime}}$, is the analogous solute Rayleigh number,

$\mathrm{Q}=\frac{\mu_{e} \mathrm{H}^{2} \mathrm{~d}^{2}}{4 \pi v \rho_{0} \eta}$, is the Chandrasekhar number,

and $T_{A}=\left(\frac{2 \Omega d^{2}}{v}\right)^{2}$, is the Taylor number.

Here we assume that the temperature at the boundaries is kept fixed, the fluid layer is confined between two boundaries and adjoining medium is electrically non conducting. The boundary conditions appropriate to the problem are [Chandrasekhar, (1981); Veronis, (1965)]

$$
\mathrm{W}=\mathrm{D}^{2} \quad \mathrm{~W}=\mathrm{DZ}=\Gamma=\Theta=0 \text { at } z=0 \text { and } 1,
$$

and the components of $h$ are continuous. Since the components of the magnetic field are continuous and the tangential components are zero outside the fluid, we have

$$
\mathrm{DK}=0 \text {, }
$$

on the boundaries. Using the boundary conditions (48) and (49), we can show that all the even order derivatives of $W$ must vanish for $z=0$ and $z=1$ and hence, the proper solution of equation (47) characterizing the lowest mode is

$$
W=W_{0} \sin \pi z ; W_{0} \quad \text { is a constant. }
$$


Substituting equation (50) in (47), we obtain the dispersion relation

$$
\begin{aligned}
R_{1} x \lambda= & {\left[\frac{i \sigma_{1}}{\epsilon}\left(1+\frac{M}{1+\tau_{1} \pi^{2} i \sigma_{1}}\right)+\frac{1+F \pi^{2} i \sigma_{1}}{P}\right](1+x) } \\
& \left(1+x+E_{1} p_{1} i \sigma_{1}\right)\left(\frac{1+\tau_{1} \pi^{2} i \sigma_{1}}{B+\tau_{1} \pi^{2} i \sigma_{1}}\right) \\
& +\frac{S_{1} x \lambda\left(1+x+E_{1} p_{1} i \sigma_{1}\right)}{\left(D^{2}-a^{2}-E_{1}^{\prime} p_{1}^{\prime} \sigma\right)}\left(\frac{B^{\prime}+\tau_{1} \pi^{2} i \sigma_{1}}{B+\tau_{1} \pi^{2} i \sigma_{1}}\right) \\
& +\frac{Q_{1}}{\epsilon} \frac{(1+x)\left(1+x+E_{1} p_{1} i \sigma_{1}\right)}{1+x+p_{2} i \sigma_{1}}\left(\frac{1+\tau_{1} \pi^{2} i \sigma_{1}}{B+\tau_{1} \pi^{2} i \sigma_{1}}\right) \\
& +\frac{\frac{T_{A_{1}}}{\epsilon^{2}}\left(1+x+E_{1} p_{1} i \sigma_{1}\right)}{\frac{i \sigma_{1}}{\epsilon}\left(1+\frac{M}{1+\tau_{1} \pi^{2} i \sigma_{1}}\right)+\frac{1-F \pi^{2} i \sigma_{1}}{P}}\left(\frac{1+\tau_{1} \pi^{2} i \sigma_{1}}{B+\tau_{1} \pi^{2} i \sigma_{1}}\right),
\end{aligned}
$$

where $R_{1}=\frac{R}{\pi^{4}}, S_{1}=\frac{S}{\pi^{4}}, T_{A_{1}}=\frac{T_{A}}{\pi^{4}}, x=\frac{a^{2}}{\pi^{2}}, i \sigma_{1}=\frac{\sigma}{\pi^{2}}, P=\pi^{2} P_{l}, Q_{1}=\frac{Q}{\pi^{4}}$.

Equation (51) is required dispersion relation accounting for the effect of suspended particles, stable solute gradient, magnetic field, medium permeability, variable gravity field, rotation on thermosolutal instability of Rivlin-Ericksen elastico-viscous fluid in porous medium.

\section{$5 \quad$ Stability of the system and oscillatory modes}

Here we examine the possibility of oscillatory modes, if any, in Rivlin-Ericksen elastico-viscous fluid due to the presence of suspended particles, stable solute gradient, rotation, magnetic field, viscoelasticity and variable gravity field. Multiply equation (40) by $W^{*}$ the complex conjugate of $W$, integrating over the range of $\mathrm{z}$ and making use of equations (41)-(44) with the help of boundary conditions (48) and (49), we obtain

$$
\begin{aligned}
& {\left[\frac{\sigma}{\epsilon}\left(1+\frac{M}{1+\tau_{1} \sigma}\right)+\frac{1+F \sigma}{P_{l}}\right] I_{1}-\frac{\mu_{e} \epsilon \eta}{4 \pi v \rho_{0}} \frac{1+\tau_{1} \sigma^{*}}{B+\tau_{1} \sigma^{*}}\left(I_{2}+p_{2} \sigma^{*} I_{3}\right)} \\
& \quad-\frac{\alpha a^{2} \lambda g_{0} k}{v \beta} \frac{1+\tau_{1} \sigma^{*}}{B+\tau_{1} \sigma^{*}}\left(I_{4}+E_{1} p_{1} \sigma^{*} I_{5}\right) \\
& \quad+d^{2}\left[\frac{\sigma^{*}}{\epsilon}\left(1+\frac{M}{1+\tau_{1} \sigma}\right)+\frac{1+F \sigma^{*}}{P_{l}}\right] I_{6}
\end{aligned}
$$




$$
\begin{aligned}
& +\frac{\mu_{e} \in \eta d^{2}}{4 \pi v \rho_{0}} \frac{1+\tau_{1} \sigma^{*}}{B+\tau_{1} \sigma^{*}}\left(I_{7}+p_{2} \sigma^{*} I_{8}\right) \\
& +\frac{\alpha^{\prime} a^{2} \lambda g_{0} \kappa^{\prime}}{v \beta^{\prime}} \frac{1+\tau_{1} \sigma^{*}}{B^{\prime}+\tau_{1} \sigma^{*}}\left(I_{9}+E_{1}^{\prime} p_{1}^{\prime} \sigma^{*} I_{10}\right)=0,
\end{aligned}
$$

where

$$
\begin{aligned}
& \mathrm{I}_{1}=\int_{0}^{1}\left(|\mathrm{DW}|^{2}+\mathrm{a}^{2}|\mathrm{~W}|^{2}\right) \mathrm{d} z, \\
& \mathrm{I}_{2}=\int_{0}^{1}\left(\left|\mathrm{D}^{2} \mathrm{~K}\right|^{2}+\mathrm{a}^{4}|\mathrm{~K}|^{2}+2 \mathrm{a}^{2}|\mathrm{DK}|^{2}\right) \mathrm{d} z, \\
& \mathrm{I}_{3}=\int_{0}^{1}\left(|\mathrm{DK}|^{2}+\mathrm{a}^{2}|\mathrm{~K}|^{2}\right) \mathrm{d} z, \\
& \mathrm{I}_{4}=\int_{0}^{1}\left(|\mathrm{D} \Theta|^{2}+\mathrm{a}^{2}|\Theta|^{2}\right) \mathrm{d} z, \\
& \mathrm{I}_{5}=\int_{0}^{1}|\Theta|^{2} \mathrm{~d} z, \\
& \mathrm{I}_{6}=\int_{0}^{1}|\mathrm{Z}|^{2} \mathrm{~d} z, \\
& \mathrm{I}_{7}=\int_{0}^{1}\left(|\mathrm{DX}|^{2}+\mathrm{a}^{2}|\mathrm{X}|^{2}\right) \mathrm{d} z, \\
& \mathrm{I}_{8}=\int_{0}^{1}|\mathrm{X}|^{2} \mathrm{~d} z \\
& \mathrm{I}_{9}=\int_{0}^{1}\left(|\mathrm{D} \Gamma|^{2}+\mathrm{a}^{2}|\Gamma|^{2}\right) \mathrm{d} z, \\
& \mathrm{I}_{0}
\end{aligned}
$$

The integral parts $I_{1}-I_{10}$ are all positive definite. Putting $\sigma=i \sigma_{i}$ in equation (52), where $\sigma_{i}$ is real and equating the imaginary parts, we obtain 


$$
\begin{aligned}
& {\left[\frac{1}{\epsilon}\left(1+\frac{M}{1+\tau_{1}^{2} \sigma_{i}^{2}}\right)+\frac{F}{P_{l}}\right]\left(I_{1}-d^{2} I_{4}\right) \sigma_{i}} \\
& -\frac{\mu_{e} \epsilon \eta}{4 \pi v \rho_{0}}\left[\left(\frac{\tau_{1}(B-1)}{B^{2}+\tau_{1}^{2} \sigma_{i}^{2}}\right) I_{2}+\frac{B+\tau_{1}^{2} \sigma_{i}^{2}}{B^{2}+\tau_{1}^{2} \sigma_{i}^{2}} p_{2} I_{3}\right] \sigma_{i} \\
& +\frac{\alpha a^{2} \lambda g_{0} k}{v \beta}\left[\left(\frac{\tau_{1}(B-1)}{B^{2}+\tau_{1}{ }^{2} \sigma_{i}{ }^{2}}\right) I_{4}+\frac{B+\tau_{1}{ }^{2} \sigma_{i}{ }^{2}}{B^{2}+\tau_{1}{ }^{2} \sigma_{i}{ }^{2}} E_{1} p_{1} I_{5}\right] \sigma_{i} \\
& +\frac{\alpha^{\prime} a^{2} \lambda g_{0} K^{\prime}}{v \beta^{\prime}}\left[\left(\frac{\tau_{1}\left(B^{\prime}-1\right)}{B^{\prime 2}+\tau_{1}{ }^{2} \sigma_{i}{ }^{2}}\right) I_{9}+\frac{B^{\prime}+\tau_{1}{ }^{2} \sigma_{i}{ }^{2}}{B^{\prime 2}+\tau_{1}{ }^{2} \sigma_{i}{ }^{2}} E_{1}^{\prime} p_{1}^{\prime} I_{10}\right] \sigma_{i} \\
& +\frac{\mu_{e} \in \eta d^{2}}{4 \pi v \rho_{0}}\left[\left(\frac{\tau_{1}(B-1)}{B^{2}+\tau_{1}{ }^{2} \sigma_{i}{ }^{2}}\right) I_{6}+\frac{B+\tau_{1}{ }^{2} \sigma_{i}{ }^{2}}{B^{2}+\tau_{1}{ }^{2} \sigma_{i}{ }^{2}} p_{2} I_{8}\right] \sigma_{i}=0
\end{aligned}
$$

Equation (53) implies that $\sigma_{i}=0$ or $\sigma_{i} \neq 0$ which mean that modes may be non oscillatory or oscillatory. The oscillatory modes introduced due to presence of rotation, stable solute gradient, magnetic field, suspended particles, viscoelasticity and variable gravity field.

\section{The stationary convection}

For stationary convection putting $\sigma=0$ in equation (51) reduces it to

$$
\mathrm{R}_{1}=\frac{1+x}{\lambda \times \mathrm{B}}\left[\frac{1+x}{\mathrm{P}}+\frac{\mathrm{Q}_{1}}{\epsilon}+\frac{\mathrm{T}_{\mathrm{A}_{1}}(1+x) \mathrm{P}}{\left\{\epsilon(1+x)+\mathrm{Q}_{1} \mathrm{P}\right\} \epsilon}\right]+\frac{\mathrm{S}_{1} \mathrm{~B}^{\prime}}{\mathrm{B}}
$$

which expresses the modified Rayleigh number $R_{1}$ as a function of the dimensionless wave number $x$ and the parameters $T_{A_{1}}, B, P, Q_{1}$ and Rivlin-Ericksen elastico-viscous fluid behave like an ordinary Newtonian fluid since elasticoviscous parameter $F$ vanishes with $\sigma$.

To study the effects of suspended particles, rotation and medium permeability, we examine the behavior of $\frac{d R_{1}}{d B}, \frac{d R_{1}}{d T_{A_{1}}}, \frac{d R_{1}}{d Q_{1}}, \frac{d R_{1}}{d S_{1}}$ and $\frac{d R_{1}}{d P}$ analytically.

Equation (54) yields

$$
\frac{d R_{1}}{d B}=-\frac{1+x}{\lambda x B^{2}}\left[\frac{1+x}{P}+\frac{Q_{1}}{\epsilon}+\frac{T_{A_{1}}(1+x) P}{\left\{\epsilon(1+x)+Q_{1} P\right\} \epsilon}\right]-\frac{S_{1} B^{\prime}}{B^{2}},
$$

which is negative implying thereby that the effect of suspended particles is to destabilize the system when the gravity increases upward from its value go 
(i.e., $\lambda>0$ ). This stabilizing effect is an agreement with the earlier work of Scanlon and Segel [13] and Rana and Kumar [11].

From equation (54), we get

$$
\frac{d R_{1}}{d T_{A_{1}}}=\left(\frac{1+x}{\lambda x B}\right) \frac{(1+x) P}{\left\{\epsilon(1+x)+Q_{1} P\right\} \epsilon},
$$

which shows that rotation has stabilizing effect on the system when gravity increases upwards from its value $g_{0}$ (i.e., $\lambda>0$ ). This stabilizing effect is an agreement of the earlier work of Sharma and Rana [17], Rana and Kango [10].

From equation (54), we get

$$
\frac{d R_{1}}{d Q_{1}}=\frac{1+x}{\lambda x B}\left[\frac{1}{\epsilon}-\frac{T_{A_{1}}(1+x) P^{2}}{\left\{\epsilon(1+x)+Q_{1} P\right\}^{2} \epsilon}\right],
$$

which implies that magnetic field stabilizes the system, if

$$
\left\{\epsilon(1+x)+Q_{1} P\right\}^{2}>T_{A_{1}}(1+x) P^{2},
$$

and destabilizes the system, if

$$
\left\{\epsilon(1+x)+Q_{1} P\right\}^{2}<T_{A_{1}}(1+x) P^{2},
$$

when gravity increases upwards from its value $g_{0}$ (i.e., $\lambda>0$ ).

In the absence of rotation, magnetic field has destabilizing effect on the system, when gravity increases upwards from its value go (i.e., $\lambda>0$ ). From equation (54), we get

$$
\frac{\mathrm{dR}_{1}}{\mathrm{dS}_{1}}=\frac{\mathrm{B}^{\prime}}{\mathrm{B}},
$$

which is positive implying thereby that the stable solute gradient has a stabilizing effect. This stabilizing effect is an agreement of the earlier work of Sharma and Rana [17],

It is evident from equation (54) that

$$
\frac{d R_{1}}{d P}=-\frac{(1+x)^{2}}{\lambda x B}\left[\frac{1}{P^{2}}-\frac{T_{A_{1}}(1+x)}{\left\{\epsilon(1+x)+Q_{1} P\right\}^{2}}\right],
$$

From equation (58), we observe that medium permeability has destabilizing effect when $\left\{\epsilon(1+x)+Q_{1} P\right\}^{2}<T_{A_{1}}(1+x) P^{2}$ and medium permeability has a stabilizing effect when $\left\{\epsilon(1+x)+Q_{1} P\right\}^{2}>T_{A_{1}}(1+x) P^{2}$, when gravity increases upwards from its value $g_{0}$ (i.e.,$\lambda>0$ ). 
In the absence of rotation and for constant gravity field $\frac{d R_{1}}{d P}$ is always negative implying thereby the destabilizing effect of medium permeability which is identical with the result as derived by Rana and Kumar [11], Rana and Kango [10].

The dispersion relation (54) is analyzed numerically. Graphs have been plotted by giving some numerical values to the parameters, to depict the stability characteristics.

In Fig. 1, Rayleigh number $R_{1}$ is plotted against suspended particles $B$ for $\lambda=2, \mathrm{~T}_{\mathrm{A}_{1}}=5, \epsilon=0.5, \mathrm{P}=0.2, \mathrm{Q}_{1}=10, \mathrm{~S}_{1}=10, \mathrm{~B}^{\prime}=2$ for fixed wave numbers $x=0.2, x=0.5$, and $x=0.8$. For the wave numbers $x=0.2, x=0.5$, and $x=0.8$, suspended particles have a destabilizing effect.

In Fig. 2, Rayleigh number $R_{1}$ is plotted against rotation $T_{A_{1}}$ for $B=3$, $\lambda=2, \epsilon=0.2, P=0.2, Q_{1}=10, S_{1}=10, B^{\prime}=2$ for fixed wave numbers $x=0.2, x=0.5$, and $x=0.8$. This shows that rotation has a stabilizing effect for fixed wave numbers $x=0.2, x=0.5$, and $x=0.8$.

In Fig. 3, Rayleigh number $R_{1}$ is plotted magnetic field $Q_{1}$ for $B=3$, $\lambda=2, \epsilon=0.2, \mathrm{~T}_{\mathrm{A}_{1}}=5, \mathrm{P}=0.2, \mathrm{~S}_{1}=10, \mathrm{~B}^{\prime}=2$ for fixed wave numbers $x=0.2, x=0.5$, and $x=0.8$. This shows that magnetic field has a destabilizing effect for $\mathrm{Q}_{1}=0.1$ to 1.5 and has a stabilizing effect for $\mathrm{Q}_{1}=1.5$ to 10 .

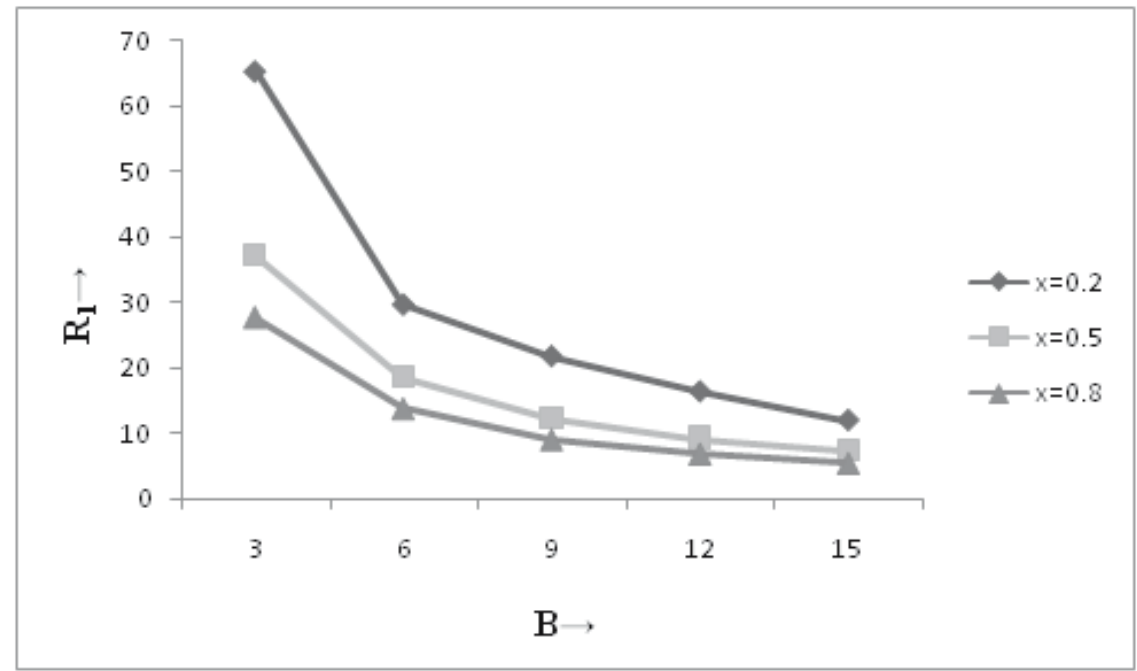

Figure 1: Variation of Rayleigh number $R_{1}$ with suspended particles $B$ for $\lambda=2, \mathrm{~T}_{\mathrm{A}_{1}}=5, \mathrm{Q}_{1}=10, \epsilon=0.2, \mathrm{P}=0.2, \mathrm{~S}_{1}=10, \mathrm{~B}^{\prime}=2$ for fixed wave numbers $x=0.2, x=0.5$, and $x=0.8$. 


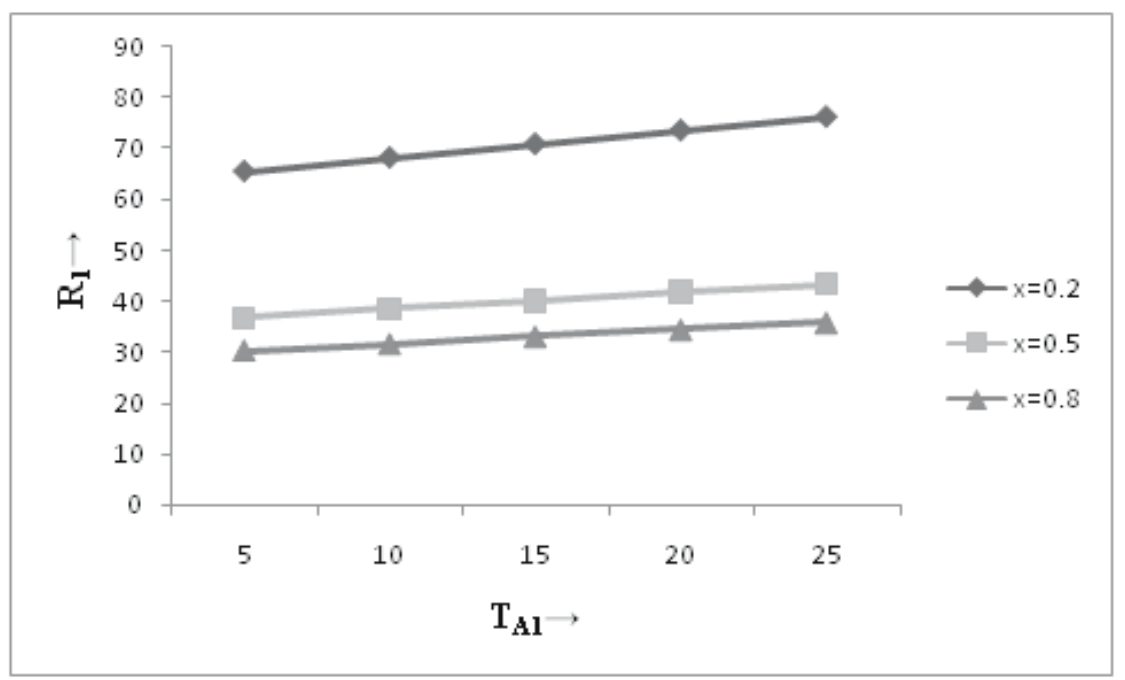

Figure 2: Variation of Rayleigh number $R_{1}$ with magnetic field $S_{1}$ for $B=3$, $\lambda=2, \epsilon=0.2, P=0.2, T_{A_{1}}=5, Q_{1}=10$, for fixed wave numbers $x=0.2, x=$ 0.5 , and $x=0.8$.

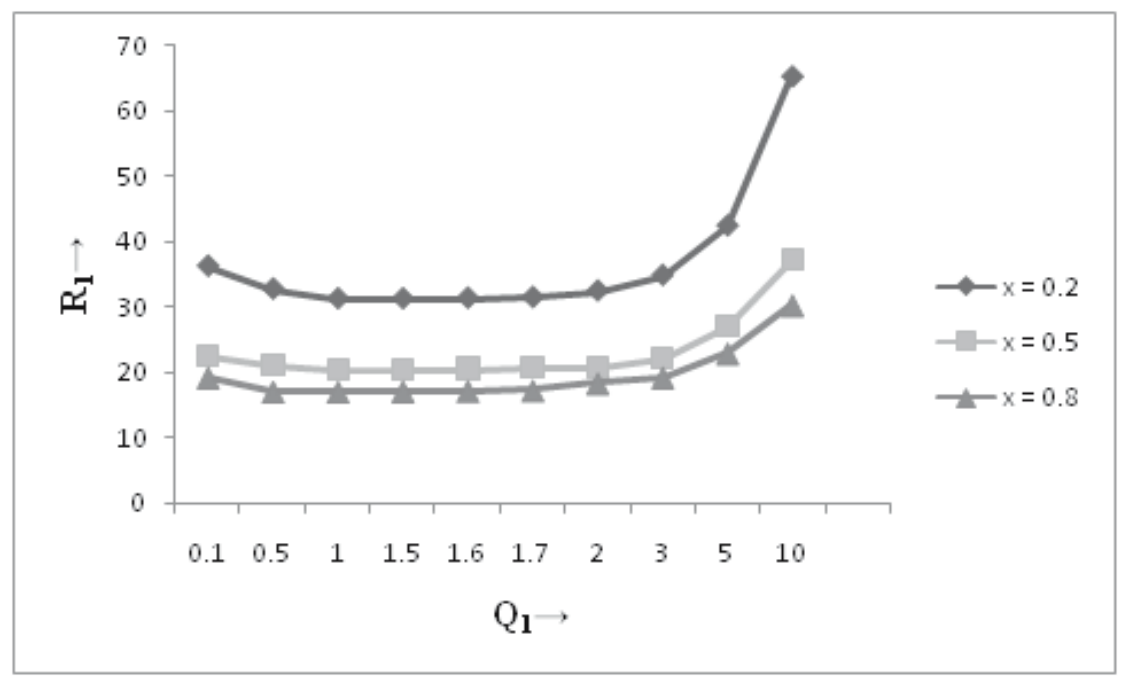

Figure 3: Variation of Rayleigh number $R_{1}$ with magnetic field $Q_{1}$ for $B=$ $3, \lambda=2, \epsilon=0.2, P=0.2, T_{A_{1}}=5, S_{1}=10, B^{\prime}=2$ for fixed wave numbers $x=0.2, x=0.5$, and $x=0.8$. 


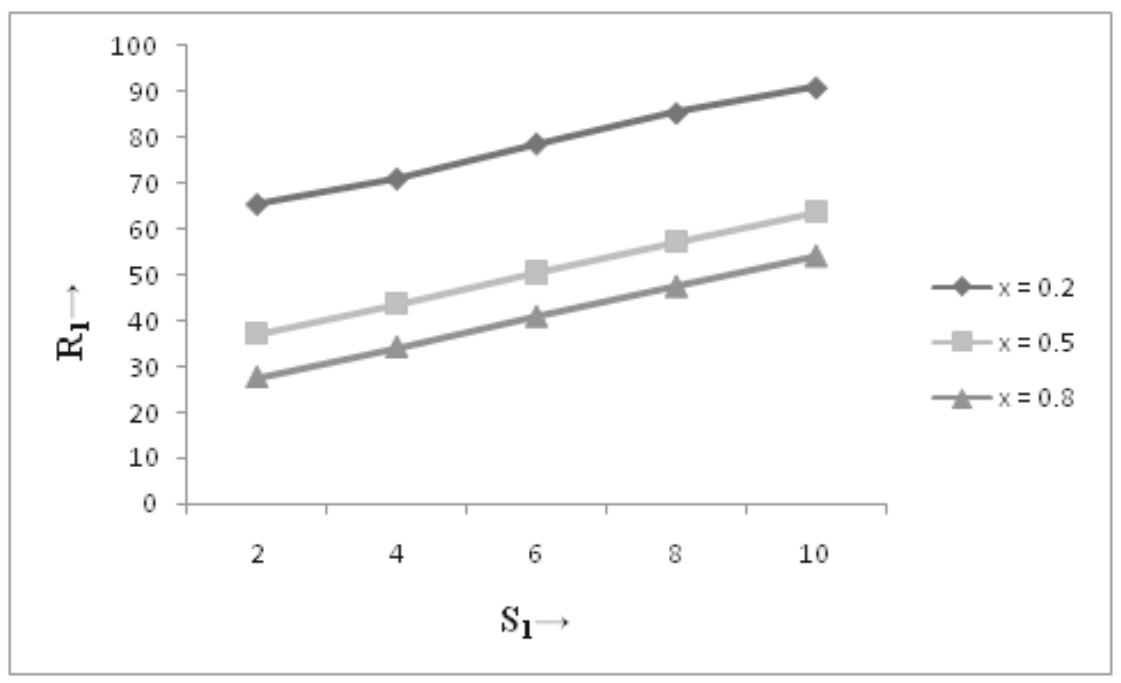

Figure 4: Variation of Rayleigh number $R_{1}$ with magnetic field $S_{1}$ for $B=3, \lambda=$ $2, \epsilon=0.2, P=0.2, T_{A_{1}}=5, Q_{1}=10$ for fixed wave numbers $x=0.2, x=0.5$, and $x=0.8$.

In Fig. 4, Rayleigh number $R_{1}$ is plotted against stable solute gradient $\mathrm{B}^{\prime}$ for $B=3, \lambda=2, \epsilon=0.2, P=0.2, Q_{1}=10, S_{1}=10$, for fixed wave numbers $x=0.2, x=0.5$, and $x=0.8$. This shows that the stable solute gradient has a stabilizing effect for fixed wave numbers $x=0.2, x=0.5$ and $x=0.8$.

In Fig. 5, Rayleigh number $\mathrm{R}_{1}$ is plotted against medium permeability $\mathrm{P}$ for $\mathrm{B}=3, \lambda=2, \epsilon=0.2, \mathrm{~T}_{\mathrm{A}_{1}}=5, \mathrm{Q}_{1}=2, \mathrm{~S}_{1}=10, \mathrm{~B}^{\prime}=2$ for fixed wave numbers $x=0.2, x=0.5$, and $x=0.8$. This shows that medium permeability has a destabilizing effect for $\mathrm{P}=0.1$ to 0.8 and has a stabilizing effect for $\mathrm{P}=0.8$ to 2.0 .

\section{Conclusion}

The thermosolutal instability of Rivlin-Ericksen elastico-viscous rotating fluid permeated with suspended particles and variable gravity field in porous medium in hydromagnetics has been investigated. For the stationary convection, it has been found that the rotation has stabilizing effect on the system as gravity increases upward from its value go (i.e. for $\lambda>0$ ). The stable solute gradient has stabilizing effect on the system and is independent of gravity field. The suspended particles are found to have destabilizing effect on the system 


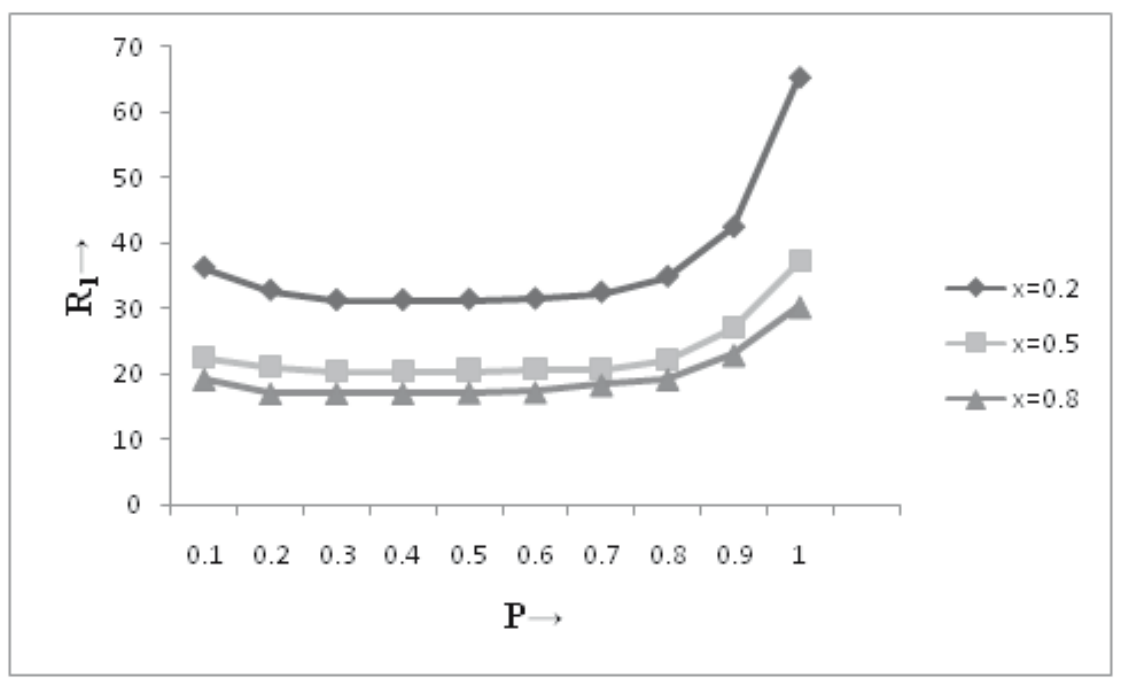

Figure 5: Variation of Rayleigh number $\mathrm{R}_{1}$ with medium permeability $\mathrm{P}$ for $\mathrm{B}=3, \lambda=2, \mathrm{Q}_{1}=2, \epsilon=0.2, \mathrm{~T}_{\mathrm{A}_{1}}=5, \mathrm{~S}_{1}=10, \mathrm{~B}^{\prime}=2$ for fixed wave numbers $x=0.2, x=0.5$ and $x=0.8$.

as gravity increases upward from its value go (i.e. for $\lambda>0$ ) whereas the medium permeability has a stabilizing / destabilizing effect on the system for $\left\{\epsilon(1+x)+Q_{1} P\right\}^{2}<T_{A_{1}}(1+x) P^{2} /\left\{\epsilon(1+x)+Q_{1} P\right\}^{2}>T_{A_{1}}(1+x) P^{2}$, as gravity increases upward from its value go (i.e. for $\lambda>0$ ). The magnetic field has stabilizing destabilizing effecton the system for $\left\{\epsilon(1+x)+Q_{1} P\right\}^{2}>$ $\mathrm{T}_{\mathrm{A}_{1}}(1+x) \mathrm{P}^{2} /\left\{\epsilon(1+x)+\mathrm{Q}_{1} \mathrm{P}\right\}^{2}<\mathrm{T}_{\mathrm{A}_{1}}(1+x) \mathrm{P}^{2}$, as gravity increases upward from its value $g_{0}$ (i.e. for $\lambda>0$ ). The presence of rotation, gravity field, suspended particles and viscoelasticity introduces oscillatory modes. The effects of rotation, suspended particles and medium permeability on thermal instability have also been shown graphically.

\section{Acknowledgments}

Author would like to thanks one of the referees for his valuable comments and suggestion for the improvement of the paper. 


\section{Nomenclature}

$\begin{array}{ll}\mathbf{q} & \text { Velocity of fluid } \\ \mathrm{q}_{\mathrm{d}} & \text { Velocity of suspended particles } \\ \mathrm{p} & \text { Pressure } \\ \mathrm{g} & \text { Gravitational acceleration vector } \\ \mathrm{g} & \text { Gravitational acceleration } \\ \mathrm{k}_{1} & \text { Medium permeability } \\ \mathrm{T} & \text { Temperature } \\ \mathrm{t} & \text { Time coordinate } \\ \mathrm{c}_{\mathrm{f}} & \text { Heat capacity of fluid } \\ \mathrm{c}_{\mathrm{pt}} & \text { Heat capacity of particles } \\ \mathrm{mN} & \text { Mass of the particle per unit volume } \\ k & \text { Wave number of disturbance } \\ k_{x}, k_{y} & \text { Wave numbers in x and y directions } \\ \mathrm{p}_{1} & \text { Thermal Prandtl number } \\ \mathrm{P}_{l} & \text { Dimensionless medium permeability } \\ \mathrm{Q} & \text { Magnetic field } \\ \mathrm{T}_{\mathrm{A}} & \text { Taylor number }\end{array}$

\section{Symbols}

$\epsilon \quad$ Medium porosity

$\rho \quad$ Fluid density

$\mu \quad$ Fluid viscosity

$\mu^{\prime} \quad$ Fluid viscoelasticity

$v$ Kinematic viscosity

$v^{\prime}$ Kinematic viscoelasticity

$\eta \quad$ Particle radius

к Thermal diffusitivity

$\kappa^{\prime}$ Solute diffusivity

$\alpha$ Thermal coefficient of expansion

$\alpha^{\prime}$ Solvent coefficient of expansion

$\beta$ Adverse temperature gradient

$\beta^{\prime}$ Solute gradient

$\Theta$ Perturbation in temperature

$\mathrm{n}$ Growth rate of the disturbance 
$\delta \quad$ Perturbation in respective physical quantity

$\zeta \quad z$-component of vorticity

$\xi \quad z$-component of current density

$\Omega$ Rotation vector having components $(0,0, \Omega)$

$\gamma$ Perturbation in solute concentration

$\mu_{e} \quad$ Magnetic permeability

\section{References}

[1] P. K. Bhatia, J. M. Steiner, Thermal instability of fluid layer in Hydromagnetics, J. Math. Anal. Appl. 41 (1973), 271-289.

[2] M. K. Brakke, Zone electrophoresis of dyes, proteins and viruses in density gradient columns of sucrose solutions, Arch. Biochem. Biophys, 55 (1955), 175-190.

[3] S. Chandrasekhar, Hydrodynamic and hydromagnetic stability, Dover Publications, New York, 1981.

[4] A. Garg, R. K. Srivastava, K. K. Singh, Rectilinear oscillations of a sphere along its diameter in conducting dusty Rivlin-Ericksen fluid in the presence of magnetic field, Proc. Nat. Acad. Sci. India, 64 (1994), 355-372.

[5] E. R. Lapwood, Convection of a fluid in porous medium, Math. Proc. Cambridge Phil. Soc., 44 (1948), 508-519.

[6] P. F. Linden, Salt fingers in a steady shear flow, Geophysics Fluid Dynamics, 6 (1974), 1-27.

[7] Lister C. R. B. Lister, On the thermal balance of a mid ocean ridge, Geophysics, J. Roy. Astr. Soc., 26 (1972), 515-535.

[8] J. A. M. McDonnel, Cosmic dust, John Wiley and Sons, Toronto, 1978.

[9] G. K. Pradhan, P. C. Samal, Thermal instability of a fluid layer under variable body forces, J. Math. Anal. Appl., 122 (1987), 487-498.

[10] G. C. Rana, S. K. Kango, Thermal instability of compressible Walters' (Model B') elastico-viscous rotating fluid a permeated with suspended dust particles in porous medium, Advances in Applied Science Research, 2 (2011), 586-597. 
[11] G. C. Rana, S. Kumar, Thermal instability of a Rivlin-Ericksen elasticoviscous rotating fluid permeated with suspended particles and variable gravity field in porous medium, Studia Geotechnica et Mechanica, XXXII (2010), 39-54.

[12] R. S. Rivlin, J. L. Ericksen, Stress deformation relations for isotropic materials, J. Rational Mech. Anal., 4 (1955), 323-334.

[13] J. W. Scanlon, L. A. Segel, Effect of suspended particles on onset of Be'nard convection, Physics Fluids, 16 (1973), 1573-78.

[14] R. C. Sharma, Effect of rotation on thermal instability of a viscoelastic fluid, Acta Physica Hungarica, 40 (1975), 11-17.

[15] R. C. Sharma, Sunil, Thermal instability of an Oldroydian viscoelastic fluid with suspended particles in hydromagnetics in porous medium, $J$. of Polymer Plastic Technology and Engineering, 33 (1994), 323-339.

[16] V. Sharma, G. C. Rana, Thermal instability of a Walters' ((Model B') elastico-viscous fluid in the presence of variable gravity field and rotation in porous medium, J. Non-Equilib. Thermodyn., 26 (2001), 31-40.

[17] V. Sharma, G. C. Rana, Thermosolutal instability of a Walters' ((Model $\mathrm{B}^{\prime}$ ) elastico-viscous rotating fluid in the presence of magnetic field and variable gravity field in porous medium, Proc. Nat. Acad. Sci. India, 73 (2003), 93-111.

[18] R. K. Srivastava, K. K. Singh, Unsteady flow of a dusty elastic-viscous Rivlin-Ericksen fluid through channels of different cross-sections in the presence of time-dependent pressure gradient, Bull. Calcutta Math. Soc., 80 (1988), 286-308.

[19] H. Stomel, K. N. Fedorov, Small scale structure in temperature and salinity near Timor and Mindanao, Tellus, 19 (1967), 306-325.

[20] G. Veronis, On finite amplitude instability in thermohaline convection, $J$. Marine Res., 23 (1967), 1-17.

[21] R. A. Wooding, Rayleigh instability of a thermal boundary layer in flow through a porous medium, J. Fluid Mech., 9 (1960), 183-192. 